\title{
Salmonella enterica serotype Typhi infections in a Canadian pediatric hospital: a retrospective case series
}

\author{
Jeannette L. Comeau MD MSc, Thai Hoa Tran MD, Dorothy L. Moore MD PhD, Chi-Minh Phi MD, \\ Caroline Quach MD MSc
}

\section{Abstract}

Background: In developed countries, typhoid fever generally occurs in travellers or recent immigrants from endemic areas. Our aim was to describe the epidemiology of Salmonella enterica serotype Typhi infections among children presenting to a pediatric teaching hospital in Montréal, Quebec.

Methods: We included all patients less than 18 years of age who presented to the Montreal Children's Hospital between 1991 and 2011 with a laboratory-confirmed diagnosis of typhoid fever (Salmonella enterica ser. Typhi isolated from blood or stool) in a retrospective case series.

Results: During the study period, we identified 39 cases of typhoid fever (mean age of patient $7.5 \mathrm{yr}$ ). Four (10.3\%) of these cases occurred in newly arrived immigrants shortly after their arrival in Canada (median $15.5 \mathrm{~d}$ ). Most cases (76.9\%) occurred in children who had visited friends and relatives in their home country. None of the travellers had received a vaccination against typhoid fever before their departure. All cases presented with high fever (mean temperature $40.4^{\circ} \mathrm{C}$ ) that lasted for a mean of 15.8 days. Common accompanying symptoms included anorexia, abdominal pain, vomiting and diarrhea. All 39 isolates of $S$. enterica ser. Typhi were susceptible to third-generation cephalosporins, and 7 were resistant to ciprofloxacin. Ampicillin resistance occurred in 10 (25.6\%) of the isolates. No deaths occurred among the study participants.

Interpretation: Most cases of typhoid fever occurred in children who had travelled to endemic areas to visit friends and relatives. Thus, there is a role for increased awareness on the part of family physicians and pediatricians caring for these children to discuss travel-related infections during regularly scheduled appointments, because parents might not consult travel clinics or discuss their travel plans before travelling back to their home country.

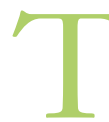
yphoid fever is a systemic febrile illness caused by the bacterium Salmonella enterica serotype Typhi that infects about 26.9 million people annually and is responsible for more than 200000 deaths each year worldwide. ${ }^{1,2}$ Overcrowded populations and poor hygienic conditions predispose developing countries to being endemic for typhoid fever, with the highest incidence found in the Indian subcontinent and Southeast Asia. ${ }^{1}$ The introduction of effective antibiotic treatment has substantially reversed this lifethreatening disease into a readily treatable condition. However, the emergence of multidrug-resistant strains of the bacterium in recent years, the lack of rapid diagnostic tests and increasing globalization have led to new challenges in the management of typhoid fever as a serious global public health concern.

Early studies showed that children and young adults between the ages of 5 and 25 years were the most likely group to be admitted to hospital for typhoid fever; ${ }^{3,4}$ thus, it was thought to be a disease predominantly affecting school-aged and adolescent children. In 1999, however, a large cohort study including more than 8000 people in Kalkaji, Delhi, India, showed that $44 \%$ of culture-positive cases of typhoid fever occurred in children less than 5 years of age. ${ }^{5}$ More recently, an Indian study profiling children in hospital in Chennai, India, showed that almost $50 \%$ of patients in hospital with typhoid fever were 5 years old or younger. ${ }^{6}$

In developed countries, this infection is generally seen in travellers or recent immigrants from endemic areas. Considering the population served by our hospital, the objective of our study was to describe the epidemiology of $S$. enterica ser. Typhi

Competing interests: Caroline Quach has a grant pending from GlaxoSmithKline to study rotavirus. No other competing interests were declared.

This article has been peer reviewed.

Correspondence to: Caroline Quach, caroline.quach @ mcgill.ca CMAJ Open 2013.DOI:10.9778/cmajo.20120012 
infections among children presenting to the Montreal Children's Hospital from 1991 to 2011. Our aim was to improve preventive strategies for this infection.

\section{Methods}

\section{Study setting}

Montréal, Quebec, is an economically and culturally diverse urban centre. Its metropolitan area has a population of about 1.8 million people, an estimated $28 \%$ of whom are international immigrants. ${ }^{7}$ More than 120 ethnic communities are represented in this population, and statistics from 2006 reported that most newly arrived immigrants came from Asia (31.1\%) and Africa (27.8\%). ${ }^{7}$ The Montreal Children's Hospital is a pediatric tertiary care centre affiliated with the McGill University Health Centre. The hospital is in downtown Montréal and receives about 5000-6500 emergency department visits per month.

\section{Study population}

We included all patients less than 18 years of age who presented to the hospital with a laboratory-confirmed diagnosis of S. enterica ser. Typhi infection from January 1991 to December 2011. We defined a case as a culture from blood or stool positive for $S$. enterica ser. Typhi. We identified cases from the database for the hospital's microbiology laboratory. Serology was not performed.

\section{Study design}

We performed a retrospective chart review for all identified patients. We used standard data extraction forms to collect the following patient data: demographic characteristics (date of birth, sex, postal code of residence), country of origin, travel destination and vaccination status (including pretravel counselling, if available). We also collected data on the patients' clinical presentations and course of illness, antimicrobial susceptibility profiles of the isolates, and treatments and outcomes. Owing to changes in laboratory standards, susceptibility profiles changed over time. All available data were recorded.

Ethics approval was obtained from the Director of Professional Services at the Montreal Children's Hospital before we began this chart review.

\section{Statistical analysis}

We performed univariate analysis using descriptive statistics (mean, median and standard deviation), relative risk with $95 \%$ confidence intervals (CIs) and $t$ tests.

\section{Results}

During the study period, we identified 39 children who presented to the Montreal Children's Hospital with typhoid fever. The patients' ages ranged from 10 months to 17.7 years (mean $7.5[ \pm 4.8]$ yr) (Table 1). Five children were less than 2 years of age. The annual number of cases varied between 0 and 6 (Figure 1).
Twenty-seven patients (69.2\%) were born in Canada, whereas $12(30.8 \%)$ were born abroad but lived in Canada at the time of presentation (Table 1). Patients who had immigrated to Canada had arrived a mean of $4.9( \pm 5.6)$ years before their illness began (median $3.0 \mathrm{yr}$ [range $2 \mathrm{~d}$ to $13.5 \mathrm{yr}$ ]) (Table 1$)$. Thirty $(76.9 \%)$ patients had travelled abroad before their infection (Table 1), and 4 patients $(10.2 \%)$ were recently landed immigrants or refugees from endemic areas. A date of arrival in Canada was missing for 2 patients who had immigrated to Canada, and 3 patients had no recorded travel outside of Canada (although 1 patient had lived in Inujivik in northern Quebec, where typhoid fever has been documented). ${ }^{8}$ Newly arrived immigrants and refugees had been in Canada for a mean of 16.3 [ \pm 13.1 ] days before illness began (median 15.5 [range 2-32] d). The most common travel destinations were Bangladesh (33.3\%), India (26.7\%), Pakistan (16.7\%) and Lebanon (6.7\%) (Table 1). Travel to Bahrain, Ghana, Haiti, Jordan and Peru was also reported. The median duration of these trips was 90 (range 14-912) days. Of note, only 1 child took malaria prophylaxis while travelling, and none of the patients had

Table 1: Baseline characteristics of pediatric patients with positive results on culture for $S$. enterica ser. Typhi

\begin{tabular}{|c|c|}
\hline Characteristic & $\begin{array}{c}\text { No. }(\%)^{*} \\
n=39\end{array}$ \\
\hline Female sex & $19(48.7)$ \\
\hline Age, yr, mean \pm SD & $7.5 \pm 4.8$ \\
\hline \multicolumn{2}{|l|}{ Country of origin } \\
\hline Canada & 27 (69.2) \\
\hline Bangladesh & $6(15.4)$ \\
\hline India & $3(7.7)$ \\
\hline Bahrain & $1 \quad(2.6)$ \\
\hline Japan (arrived via Bangladesh) & $1 \quad(2.6)$ \\
\hline Pakistan & $1 \quad(2.6)$ \\
\hline $\begin{array}{l}\text { Time in Canada before presentationt (if born } \\
\text { outside Canada), yr, median } \pm S D\end{array}$ & $4.9 \pm 5.6$ \\
\hline Travel abroad before presentation & $30(76.9)$ \\
\hline \multicolumn{2}{|l|}{ Travel destination } \\
\hline Bahrain & 1 (3.3) \\
\hline Bangladesh & $10(33.3)$ \\
\hline Ghana & 1 (3.3) \\
\hline Haiti & 1 (3.3) \\
\hline India & $8(26.7)$ \\
\hline Jordan & 1 (3.3) \\
\hline Lebanon & $2(6.7)$ \\
\hline Pakistan & $5(16.7)$ \\
\hline Peru & 1 (3.3) \\
\hline Duration of trip, $d$, median (range) & $90(14-912)$ \\
\hline Malaria prophylaxis & $1 \quad(2.6)$ \\
\hline Record of typhoid vaccination & $0 \quad(0.0)$ \\
\hline $\begin{array}{l}\text { Note: SD = standard deviation. } \\
\text { *Unless stated otherwise stated. } \\
\text { †Missing data for } 2 \text { patients. }\end{array}$ & \\
\hline
\end{tabular}


a record of having received a vaccination against typhoid fever before departure (Table 1).

Children presented $12.6[ \pm 11.1]$ days after the onset of illness. All of the patients presented with fever (mean temperature $40.4^{\circ} \mathrm{C}\left[ \pm 0.6^{\circ} \mathrm{C}\right]$ ) that lasted for a mean of 15.8 days (Table 2). The mean fever-clearance time after the start of antibiotics was $4.8( \pm 3.6)$ days. Other common symptoms included anorexia (69.2\%), abdominal pain (56.4\%), vomiting (48.7\%) and diarrhea (46.2\%).

Thirty-four children $(87.1 \%)$ required admission to hospital, with a mean length of stay of 8.8 [ \pm 3.3$]$ days (Table 3). Thirty-six patients $(92.3 \%)$ were given third-generation cephalosporins (33 patients were given ceftriaxone; 3 patients were given cefotaxime). Ten patients completed their course of treatment with antibiotics administered orally, as determined by antimicrobial susceptibility results. The mean duration of the total course of antibiotics was $11.8[ \pm 4.0]$ days.

Twenty-three patients received antibiotics before they presented to hospital. These included amoxicillin (5 patients), penicillin (4 patients), ceftriaxone ( 2 patients), metronidazole (2 patients), cefprozil (1 patient), ciprofloxacin (1 patient) and amoxicillin-clavulinic acid (1 patient); 1 patient received both ciprofloxacin and metronidazole; and 8 patients stated that they had taken antibiotics but did not know which ones. Eight patients did not receive antibiotics before presentation, and data were not available for the remaining 8 patients.

Thirty-six patients $(92.3 \%)$ had blood samples that grew $S$. enterica ser. Typhi on culture, and $26(66.7 \%)$ patients had stool samples that grew the organism. One child had only a stool sample taken, and 5 children had only blood samples drawn. Two children had negative results on blood sample cultures but positive results on stool sample cultures, whereas 6 children had negative stool samples but positive blood samples. Two children had stool samples taken for which the results were not available. The mean length of time during which serial daily blood cultures remained positive following the first positive blood culture was $2.3( \pm 1.3)$ days. Among the 39 isolates of S. enterica ser.
Typhi isolated from patient blood, 29 (74.3) were susceptible to ampicillin and trimethoprim-sulfamethoxazole, and all isolates were susceptible to ceftriaxone. Eight different isolates from separate patients showed antibiotic resistance to both ampicillin and trimethoprim-sulfamethoxazole. Seven isolates were resistant to ciprofloxacin. Resistance to ampicillin was more common during the latter half of the study period (4 of 21 isolates before 2000, compared with 6 of 18 isolates after 2000) (Figure 1). Tests for resistance to ciprofloxacin were only routinely done on all isolates from 2007 onward. Of the 30 isolates found before 2007, 4 showed sensitivity to ciprofloxacin; from 2007 to 2011, 7 of 9 isolates found showed resistance to the drug.

Complications after typhoid fever were reported for 15 patients $(38.5 \%)$, including hepatitis (4 patients), coagulopathy (3 patients), meningitis ( 2 patients), cholecystitis (1 patient), shock (1 patient), delirium (1 patient), sepsis requiring administration of immunoglobulin intravenously (1 patient) and relapse requiring readmission (2 patients). Only 2 children required admission to the pediatric intensive care unit (ICU) - 1 child had coagulopathy, and 1 child was admitted primarily to establish vascular access. No deaths occurred among participants. One patient needed 2 doses of immunoglobulin administered intravenously. Complications occurred in $33.3 \%$ of children under 5 years of age and in $36.8 \%$ of patients who had been febrile for more than 2 weeks. The average time to presentation after the onset of symptoms for the 15 children who had complications was 13.3 $( \pm 11.5)$ days, which is 1 day later than for children who did not have complications but not a statistically significant delay.

Aside from the patterns of antibiotic resistance, we saw no other significant changes over time.

\section{Interpretation}

Most of the cases of typhoid fever we identified during the study period $(76.9 \%)$ occurred in Canadians who had travelled to endemic areas, mainly to the Indian subcontinent

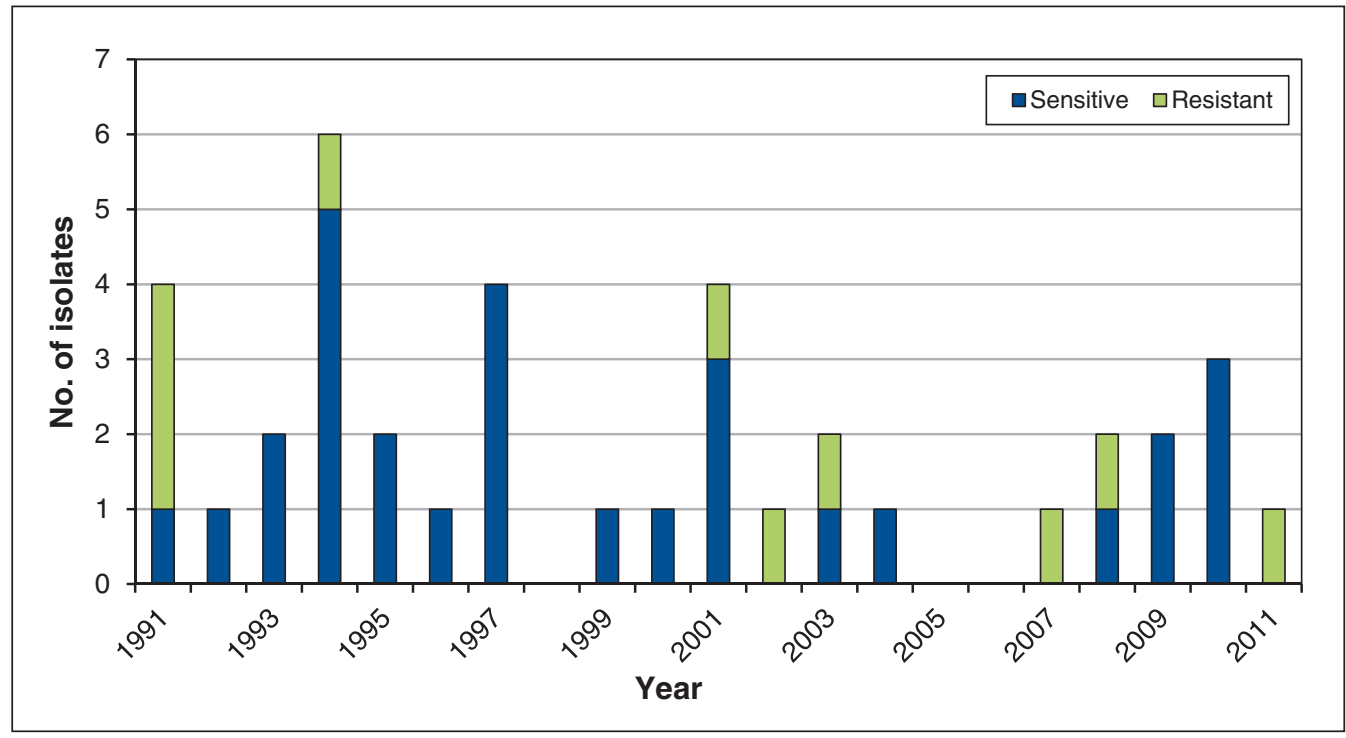

Figure 1: Distribution of cases of typhoid fever during the study period, by year sensitivity to ampicillin. 
(Bangladesh, India and Pakistan), where the incidence of the disease exceeds 700 cases per 100000 people per year. ${ }^{2}$

Although proper sanitation has greatly contributed to the near elimination of typhoid fever in developed countries, this infection remains a global public health concern. A meta-analysis of 22 population-based studies reviewed the epidemiology of typhoid fever in 2000 and found that the global burden of the disease had increased in terms of annual morbidity but decreased in mortality - from about 16 million cases and 600000 deaths in 1984, to 21650974 cases and 216510 deaths 16 years later. In North America, 453 cases of typhoid fever were recorded in $2000,{ }^{1}$ most of which occurred in returning travellers or new arrivals from endemic areas. It has been estimated that the incidence of typhoid fever among travellers to developing countries was 3-30 cases per 100000 travellers. ${ }^{1}$ Further analysis, in 2010, showed comparable findings, with an estimated worldwide incidence of 26.9 million episodes and incidence rates ranging from less than 0.1 per 100000 in Europe and Central Asia to 724.6 per 100000 in Sub-Saharan Africa and South Asia. North America (excluding Latin America) was found to have an incidence rate of 0.1 per 100000 . Mortality in the most-affected areas was found to be 7.2 per 100000 in Sub-Saharan Africa and 3.9/100 000 in South Asia. ${ }^{2}$ In addition, American data have shown that $72 \%-$ $74 \%$ of people with $S$. enterica ser. Typhi infections had a history of recent travel before their illness. ${ }^{9,10}$

Typhoid fever remains a difficult diagnosis to make clinically because of its nonspecific presentation. Consistent with current literature, the most common symptoms and signs among patients in our study were fever, anorexia, abdominal pain, diarrhea and vomiting. Constipation is often present in adults, but diarrhea is a more common clinical feature in children. ${ }^{11}$

Table 2: Clinical presentation of cases of typhoid fever in 39 pediatric patients

\begin{tabular}{|lcc|}
\hline Signs/symptoms & No. $(\%)^{*}$ \\
\hline Fever & $39(100.0)$ \\
\hline Duration, d, mean $( \pm$ SD) & $15.8 \pm 7.9$ \\
\hline Maximal temperature (average \pm SD) & $40.4 \pm 0.6$ \\
\hline Anorexia & 27 & $(69.2)$ \\
\hline Abdominal pain & 22 & $(56.4)$ \\
\hline Diarrhea & 18 & $(46.2)$ \\
\hline Vomiting & 19 & $(48.7)$ \\
\hline Headache & 15 & $(38.5)$ \\
\hline Constipation & 5 & $(12.8)$ \\
\hline Jaundice & 2 & $(5.1)$ \\
\hline Rash & 1 & $(2.6)$ \\
\hline Hepatosplenomegaly & 8 & $(20.5)$ \\
\hline Heart rate, beats/min, mean $( \pm$ SD) & $123.4 \pm 23.6$ \\
\hline Leukocyte count, cells/mL, mean $( \pm$ SD) & $8.33 \pm 4.07$ \\
\hline Platelet count cells/mL, mean $( \pm$ SD $\dagger$ & $261 \pm 285$ \\
\hline $\begin{array}{l}\text { Note: SD = standard deviation. } \\
\text { *Unless otherwise stated. } \\
\text { †Platelet range: } 71-1802, \text { median }=206\end{array}$ & & \\
\hline
\end{tabular}

The definitive diagnosis of typhoid fever relies on the isolation of S. enterica ser. Typhi from blood samples, which produce positive cultures in $60 \%-85 \%$ of cases. ${ }^{11}$ Cultures from bone marrow samples have greater sensitivity, estimated to be as high as $95 \%$, regardless of previous antibiotic treatment or duration of illness, both of which have been associated with reduced sensitivity of blood cultures. Cultures from stool samples have lower sensitivity, about $30 \%,{ }^{11}$ usually showing positive results 1 week into the course of illness. ${ }^{12}$ Our study provides further evidence of the superiority of blood over stool, with only $81.3 \%$ of patients showing positive stool cultures, compared with $94.7 \%$ of patients with positive blood cultures when tested.

The rate of complications among our patients was higher than has been reported in other studies, but the clinical presentation of the disease was milder compared with that described previously (no deaths occurred and only 2 children required admission to the pediatric ICU); ${ }^{11,13}$ previous studies have reported complications in $10 \%-15 \%$ of patients. ${ }^{11}$ In previous research, a duration of illness of more than 2 weeks was highly associated with gastrointestinal bleeding (10\%), intestinal perforation $(1 \%-3 \%)$ and typhoid encephalopathy $(10 \%-40 \%) .{ }^{11}$ This discrepancy in the rate of complications may be because $60 \%-90 \%$ of patients with S. enterica ser. Typhi infections worldwide receive treatment as outpatients, ${ }^{5,14}$ increasing the likelihood of losses to follow-up and complications not being detected.

\begin{tabular}{|c|c|}
\hline Characteristic & No. $(\%)^{*}$ \\
\hline Admission to hospital & $34(87.2)$ \\
\hline Length of stay, $d$, mean $\pm S D$ & $8.8 \pm 3.3$ \\
\hline Received antibiotics before admission & $23(59.0)$ \\
\hline \multicolumn{2}{|l|}{ In-patient treatment† } \\
\hline Ceftriaxone & $33(84.6)$ \\
\hline Cefotaxime & $3 \quad(7.7)$ \\
\hline Ampicillin & $2(5.1)$ \\
\hline Clindamycin & $1 \quad(2.6)$ \\
\hline Gentamicin & $1 \quad(2.6)$ \\
\hline Metronidazole & $1 \quad(2.6)$ \\
\hline Discharged while still taking antibiotic agent & $21(53.8)$ \\
\hline Ceftriaxone & $11(28.2)$ \\
\hline Amoxicillin & $5(12.8)$ \\
\hline Trimethoprim-sulfamethoxazole & $3 \quad(7.7)$ \\
\hline Ciprofloxacin & 1 (2.6) \\
\hline Erythromycin & $1 \quad(2.6)$ \\
\hline $\begin{array}{l}\text { Complications (shock, relapse, coagulopathy, } \neq \\
\text { cholecystitis, hepatitis) }\end{array}$ & $15(38.5)$ \\
\hline $\begin{array}{l}\text { Note: } \mathrm{SD}=\text { standard deviation. } \\
\text { *Unless otherwise stated. } \\
\text { †Some patients received more than } 1 \text { agent. } \\
\neq \text { †isseminated intravascular coagulation. }\end{array}$ & \\
\hline
\end{tabular}


None of our patients died, nor did they require surgical interventions. Recent population-based studies from South Asia suggest that children under 5 years of age had the highest incidence of complications, in contrast to previous reports from Latin America and Africa that had described a milder disease in infancy and childhood. ${ }^{6,15,16}$

The mainstay in the treatment of typhoid fever is antibiotic therapy. Historically, chloramphenicol, amoxicillin and trimethoprim-sulfamethoxazole constituted first-line therapies for uncomplicated and fully susceptible strains of the causative organism. ${ }^{17,18}$ However, the emergence of chloramphenicol-resistant typhoid fever in 1972 and the subsequent resistance to all first-line agents in the late 1980s contributed to the widespread use of fluoroquinolones. ${ }^{19}$ The efficacy of quinolones in the treatment of typhoid fever has been confirmed in multiple randomized controlled trials (RCTs) in which the cure rates exceeded $96 \%$, and the use of these agents have proven safe for all age groups, including children. ${ }^{20}$ Of note, however, we do document resistance to some fluoroquinolones in 7 of 9 isolates after 2007 (when our laboratory began routinely testing for ciprofloxacin resistance). Recently, an Australian group has shown an increase in resistance to nalidixic acid (also a fluoroquinolone) and suggests ceftriaxone or azithromycin as appropriate first-line agents. ${ }^{21}$ Our results would support this suggestion.

Most of our patients received ceftriaxone intravenously as first-line therapy. The mean fever-clearance time after the start of antibiotics in our study population was comparable with that reported in RCTs using cephalosporins. ${ }^{22-25}$ Only 2 of our patients, both of whom had received ceftriaxone, underwent relapse requiring readmission, which is a rate similar to that of $3 \%-6 \%$ reported in the literature. $13,2,2,23,26$

Because humans are the only reservoir for S. enterica ser. Typhi, control of the disease could theoretically be achieved by giving adequate antimicrobial treatment to patients with clinical cases and to asymptomatic carriers of the bacterium. However, the role of vaccination as a preventive measure is of greater importance in controlling the spread of the disease. ${ }^{27,28}$ Vaccines against typhoid fever are available and recommended for travellers to endemic areas; however, none of our patients had received the vaccine before travel. This may be due to the risk of infection upon travelling being considered benign, or simply not considered, by parents who lived in these endemic areas without becoming sick and who are returning to visit friends and relatives.

Two vaccines against typhoid recommended since 2000 by the World Health Organization both offer protection ranging from $50 \%$ to $80 \%{ }^{27}$ during the first year after vaccination: ${ }^{28}$ the live, attenuated oral vaccine Ty $21 \mathrm{a}$ and the $\mathrm{Vi}$ based polysaccharide vaccine. Both vaccines are generally well-tolerated. The Ty21a vaccine is contraindicated in pregnant women, children less than 5 years of age, patients with an acute gastrointestinal condition or inflammatory bowel disease and those who are immunocompromised. Revaccination may be repeated after 7 years have elapsed. The Vi polysaccharide vaccine is administered as a single intramuscular dose and can be given to travellers over the age of 2 years, with a booster given if more than 2-3 years have elapsed since the previous vaccination. ${ }^{29}$ Despite vaccination, routine protective measures should be practiced, such as drinking boiled or bottled water, and cooking and peeling fruits and vegetables, because immunity can be overcome if a large number of organisms are ingested. ${ }^{30}$

\section{Limitations}

Although our study was conducted at a single centre in a large metropolitan area, we were only able to identify a small number of cases. In addition, the laboratory testing for antibiotic sensitivities has changed over time, thus the resistance pattern for some antibiotics (e.g., ciprofloxacin) could not be completely established from our dataset. However, for instances in which data were only partially available, we have stated so in the text.

\section{Conclusion}

Most of the cases of typhoid fever we identified in our centre occurred in children returning from endemic areas who had not received vaccination against the disease. Although uncommon, the clinical diagnosis of typhoid fever remains difficult and should be considered in a febrile traveller. Our study shows the importance of blood and stool samples being taken from all travellers presenting with fever; stool samples alone may not always be sufficient. Furthermore, we recommend testing for sensitivity to fluoroquinolones because resistance to these agents appears to be increasing. Given this increasing resistance in various areas of the world, fluoroquinolones should not be used as first-line agents, but rather cefixime or azithromycin administered orally, or ceftriaxone or cefotaxime administered parenterally. ${ }^{31}$

The availability of 2 approved vaccines makes this disease potentially preventable for travellers. Family physicians and pediatricians should emphasize the risk of typhoid fever and other travel-related infections (e.g., malaria) to their patients, especially children whose parents are from endemic areas. This counselling, which should include discussion about safe foods and water, as well as sanitation and hygiene, may be part of regularly scheduled appointments, because parents may not seek medical advice before travelling to their home country.

\section{References}

1. Crump JA, Luby SP, Mintz ED. The global burden of typhoid fever. Bull World Health Organ 2004;82:346-53.

2. Buckle GC, Walker CL, Black RE. Typhoid fever and paratyphoid fever: Systematic review to estimate global morbidity and mortality for 2010. 7 Glob Health 2012;2:10401.

3. Stuart BM, Pullen RL. Typhoid; clinical analysis of 360 cases. Arch Intern Med (Chic) 1946;78:629-61.

4. Huckstep RL. Typhoid fever and other salmonella infections. Edinburgh (UK): E \& S Livingstone; 1962.

5. Sinha A, Sazawal S, Kumar R, et al. Typhoid fever in children aged less than 5 years. Lancet 1999;354:734-7.

6. Ganesh R, Janakiraman L, Vasanthi T, et al. Profile of typhoid fever in children from a tertiary care hospital in Chennai-South India. Indian 7 Pediatr 2010;77: $1089-92$.

7. The Montréal region at a glance. Montréal (QC): Immigration et Communautés Culturelle Québec. Available: www.immigration-quebec.gouv.qc.ca/en /settle/montreal.html (accessed 2012 Oct 2).

8. Martin D, Belanger D, Gosselin P, et al. Drinking water and potential threats to human health in Nunavik: adaptation strategies under climate change conditions. Arctic. 2007;60:7.

9. Mermin JH, Townes JM, Gerber M, et al. Typhoid fever in the United States, 
1985-1994: changing risks of international travel and increasing antimicrobial resistance. Arch Intern Med 1998;158:633-8.

10. Steinberg EB, Bishop R, Haber P, et al. Typhoid fever in travelers: who should be targeted for prevention? Clin Infect Dis 2004;39:186-91.

11. Wain J, Pham VB, Ha V, et al. Quantitation of bacteria in bone marrow from patients with typhoid fever: relationship between counts and clinical features. 7 Clin Microbiol 2001;39:1571-6.

12. Bhutta ZA. Current concepts in the diagnosis and treatment of typhoid fever. BMF 2006;333:78-82.

13. Pegues DA, Miller SI. Salmonellosis. In: Longo DL, Fauci AS, Kasper DL, et al., editors. Harrison's principles of internal medicine. 18th ed. New York (NY): McGraw-Hill; 2012. p. 1274-8.

14. Lin FY, Vo AH, Phan VB, et al. The epidemiology of typhoid fever in the Dong Thap province, Mekong Delta region of Vietnam. Am f Trop Med Hyg 2000;62: 644-8.

15. Brooks WA, Hossain A, Goswami D, et al. Bacteremic typhoid fever in children in an urban slum, Bangladesh. Emerg Infect Dis 2005;11:326-9.

16. Siddiqui FJ, Rabbani F, Hasan R, et al. Typhoid fever in children: some epidemiological considerations from Karachi, Pakistan. Int 7 Infect Dis 2006;10:215-22

17. Woodward TE, Smadel JE, Ley HL,et al. Preliminary report on the beneficial effect of chloromycetin in the treatment of typhoid fever. Ann Intern Med 1948;29:131-4

18. Kadhiravan T. Typhoid fever. In: Rakel RE, Bope ET, editors. Conn's current therapy. Philadelphia (PA): Elsevier Saunders; 2012. p. 179-81.

19. Mirza SH, Beeching NJ, Hart CA. Multi-drug resistant typhoid: a global problem. 7 Med Microbiol 1996;44:317-9.

20. Effa EE, Lassi ZS, Critchley JA, et al. Fluoroquinolones for treating typhoid and paratyphoid fever (enteric fever). Cochrane Database Syst Rev 2011; (10):CD004530.

21. Commons RJ, McBryde E, Valcanis M, et al. Twenty-six years of enteric fever in Australia: an epidemiological analysis of antibiotic resistance. Med 7 Aust 2012;196:332-6.

22. Cao XT, Kneen R, Nguyen TA, et al. A comparative study of ofloxacin and cefixime for treatment of typhoid fever in children. The Dong Nai Pediatric Center Typhoid Study Group. Pediatr Infect Dis 7 1999;18:245-8.

23. Girgis NI, Butler T, Frenck RW, et al. Azithromycin versus ciprofloxacin for treatment of uncomplicated typhoid fever in a randomized trial in Egypt that included patients with multidrug resistance. Antimicrob Agents Chemother 1999;43:1441-4.
24. Memon IA, Billoo AG, Memon HI. Cefixime: an oral option for the treatment of multidrug-resistant enteric fever in children. South Med f 1997;90:1204-7.

25. Frenck RW Jr., Nakhla I, Sultan Y, et al. Azithromycin versus ceftriaxone for the treatment of uncomplicated typhoid fever in children. Clin Infect Dis 2000; 31:1134-8.

26. Ahmad KA, Khan LH, Roshan B, et al. Factors associated with typhoid relapse in the era of multiple drug resistant strains. F Infect Dev Ctries 2011;5:727-31.

27. Guzman CA, Borsutzky S, Griot-Wenk M, et al. Vaccines against typhoid fever. Vaccine 2006;24:3804-11.

28. Fraser A, Goldberg E, Acosta CJ, et al. Vaccines for preventing typhoid fever. Cocbrane Database Syst Rev 2007 (3):CD001261.

29. Canadian immunization guide: typhoid vaccine. Ottawa (ON): Public Health Agency of Canada; 2006. Available: www.phac-aspc.gc.ca/publicat/cig-gci/p04 -typh-engphp\#ru (accessed 2012 Oct. 5).

30. Rongkavilit C. Immunization for pediatric international travelers. Pediatr Ann 2011;40:346-50.

31. Centers for Disease Control and Prevention. CDC Health Information for International Travel 202. New York (NY): Oxford University Press; 2012. Available: http://wwwnc.cdc.gov/travel/page/2012-yellow-book-about.htm (accessed 2013 Apr. 29).

Affiliations: From the Infectious Diseases Division (Comeau, Tran, Moore, Quach), Department of Pediatrics; the Department of Pediatrics (Comeau, Phi); and the Department of Microbiology (Quach), Montreal Children's Hospital, McGill University, Montréal, Que.

Contributors: Jeannette Comeau collected the data and drafted the manuscript. Thai Tran, Dorothy Moore, Chi-Minh Phi and Caroline Quach contributed to the study's design, participated in data collection and revised manuscript for important intellectual content. All of the authors approved the final version of the article submitted for publication.

Supplemental information: For reviewer comments and the original submission of this manuscript please see www.cmajopen.ca/content/1/1 /E56/supple/DC1 